\title{
FLEXIBLE SOLAR POWER TREE - A CONCEPT
}

\author{
Mukesh Kumar \\ Physics Department SSNC \\ University of Delhi, INDIA
}

\author{
J P Kesari \\ Mechanical department \\ Delhi Technological University
}

\author{
Lokesh Rana \\ Physics \& Astrophysics Department \\ University of Delhi, INDIA
}

\begin{abstract}
Sunlight is always a great asset for human beings. Among various systems available to access solar power, the most well known innovation is the photovoltaic(PV) transformation of daylight into electricity. Regardless of its benefits, sunlight based PV innovation has issues such as bulky and less efficiency. The most precious process of photosynthesis, the sun catching nature of trees is an inventive thought of light catching cells. The idea of a sun oriented flexible tree is fit for tending to these issues adequately with polish. In this paper efforts have been made to audit the parts of the sun based tree and its energy harvesting capability. The different energy enhancement structures are additionally talked about alongside utilizations of the sun powered tree. The paper additionally addresses the difficulties engaged with this innovation and proposes future research heading.
\end{abstract}

Keywords- Photovoltaic, Solar tree, Solar Energy, Renewable Energy

\section{INTRODUCTION}

The journey for green and supportable energy sources has gotten probably the greatest test for our time, because of exhaustion of fossil energy products, environmental challenges, an Earth-wide temperature boost and perpetually developing energy demands. Energy from the sun comprises light and warmth from the Sun, it is being utilized via different advancing technologies. Few of them are like sunlight based heat collectors, sun oriented photovoltaic, sun based heat power, sun based electricity powerhouse, sun-powered building design and counterfeit photosynthesis [1].

The most well-known use of the sun is electric energy generation through the photovoltaic (PV) frameworks. Sun based PV execution is reliant on the nearby climatic conditions and accessibility of sun-powered radiation. Sun based radiation appraisal and estimation is particularly useful for the appropriate structure of sun based energy change frameworks. The trap frequency and intensity of the Sun beams vacillate for the duration of the day and throughout the year. In this manner, sun-powered modules fixed at a specific point may not be completely advanced. Sun following PV frameworks can be structured, however they essentially raise the all-out expense of energy generation as they are exorbitant and require support. Another, encouraging approach to coordinate sun oriented PV into the developed condition is through multidimensional Photovoltaic frameworks [6-8]. One of the essential installation components for the establishment of all such sun based PV modules is land Rooftop sun oriented $\mathrm{PV}$ is an alluring choice, however, it has constrained space for modules to be masterminded in a cluster or multistoried building particularly inland limited urban territories. The Sun following PV frameworks can be a possible solution but they fundamentally raise the all out expense of energy as they are exorbitant and require support. Another challenge in this encouraging solar powered PV execution deals with issues like installation space requirement, low PV efficiency, approach to coordinate sun based PV frameworks etc.

Significant cost reductions have been observed in the past few decades along with high efficiency in photovoltaic based solar cells with strong increase in people acceptability. The PV technology now positioned to provide low-cost energy options and have enormous capacity for future energy generation. Improvement in energy-conversion efficiency reduces such costs, with mass manufacturing likely to bring down the additional costs associated for achieving higher efficiencies. This suggests that the industries will evolve beyond the conventional single-junction solar cells that presently dominate commercial production, where energy-conversion efficiencies are constrained by practical values of ShockleyQueisser limits to below 30\%. Contrary to that nature's solar capturing technology, plants exploit the Sun more effectively [3]. The low effectiveness is due to associated losses like spectrum loss, reflection loss and heat generation and so forth.

In this article the overall prospects for a range of approaches that can potentially exceed these limits are based on ultimate efficiency prospects, material requirements and installations outlook. The challenges involved in this technique are considered and suggestions are proposed to overcome them. Finally the concept of solar trees is discussed to overcome above losses through law of conservation and minimization process.

\section{Similarities BetweEn Solar CELl AND PHOTOSYNTHESIS}

Like plants, solar cells gather their energy from sunlight. Plant leaves collect sunlight and transform this energy into 


\section{International Journal of Engineering Applied Sciences and Technology, 2020 Vol. 5, Issue 2, ISSN No. 2455-2143, Pages 217-221 \\ Published Online June 2020 in IJEAST (http://www.ijeast.com)}

chemical energy. Similarly, photovoltaic solar cells convert the sun's energy into electricity. They perform the same function but in different senses. The main resemblance between the two is that both photovoltaic and photosynthetic plants are supposed to absorb photons.

This task of absorption of sunlight is carried out by a molecule called chlorophyll in nature (plants). In the case of photovoltaic, scientists have designed materials where the electrons can absorb the energy of the sun's photons. In both cases, photons are absorbed by electrons, which take up the extra energy and get excited to a higher energy state known as excited electron. The process of conversion of the energy absorbed by excited electrons should be fast before they lose their energy and go back to their initial state. In the case of photosynthesis, this issue is resolved by moving the electron from one molecule to another until it finally settles in a molecule that can store this energy for a long time. In photovoltaic, the excited electrons are carried off into a circuit, where they are either used to operate directly or are routed into a battery for storage [4].

There is a type of solar cell that is designed to be as analogous to photosynthesis. This is a non-conventional photovoltaic cell that tries to reproduce the way photosynthesis works. Instead of moving an electron as quickly as possible through a crystal of identical atoms, this dye-sensitized solar cell absorbs energy in a dye molecule, then transfers the excited electron into another material located adjacent to the dye molecule. This technique makes sure that the electron doesn't lose its energy ineffectively. When connected to a circuit, this electron makes its way through the second material without risking losing its energy.

\section{Plant ARChitecture InSPIRE Solar CELl DESIGN}

Plants exhibit a variety of systematic structures and structures, which are difficult to explain. Plants are dependent on the light energy they receive from photosynthesis, and strong sunlight is the most important factor in photosynthetic influence, but in addition the leaf temperature, leaf water status, and many other physiological procedures of the plant also influence photosynthesis. There are no fixed configuration rules in all conditions.

Geometrically it is determined by only two parameters: the propagation limit and the branch length. They are undoubtedly more cohesive and consistently attracted to authors of plant conditions and development models. In trees, the leaves outside the hiding place are different from those inside the tree cover. The outer leaves are called "sun leaves," and the inner leaves are "secret leaves." These leaves contain differences such as fiddle, internal structures of life, and science that enable the translation of certain energy to use different types of solar radiation more effectively. The leaves of the sun are generally normal, prolonged and larger than the secreted leaves, which contain many layers of chlorine-containing tissue and are very broad within the arteries. It is believed that the sun's leaves are better adapted to capture and use direct sunlight (if not too different to even think of causing heat and pressure related damage). Its long position is connected in parallel with the most important levels of sunlight reaching the inner cover where the foliage is located. Shaded leaves appear to make good use of sunlight, reaching into the inner shelter after exposure to different substances, such as outer leaves, in the form of direct light. Leaves of similar color can be found in an outdoor shelter such as the color towards the subtle sun. The leaf level of photosynthesis depends on the biomass of the leaf biomass as the emission of light into the leaf area. The scattering and course of leaf functioning within a tree can completely alter the effectiveness of a good harvest. The leaf area all tightened to a given plant is a very important aid that contributes to the partitioning of edible radiation. When the point from the light of the sun decreases from the equator to the highest positions, the trees of different lengths and widths have effectively changed the light capture technique. All kinds in a variety of colors to add an effort to capture a quick glow through the whole tree.

The leaf must "absorb" sunlight, water from the inside and carbon dioxide from the air that combines photosynthesis. Leaf photosynthesis, meanwhile, is influenced by these carefully regulated materials: enough sunlight and carbon dioxide for photosynthesis, yet not all that closely related heat storage or water misery. The leaves found on the Nature Trail trees are expensive and adequate or molded with a needle that is ideal for their water-conserving weather conditions and high maintenance during the day is an important way to persevere.

Plants show a striking assorted variety of structures and structures, which are hard to decipher. Plants rely upon the light energy that they catch by photosynthesis, and sunpowered radiation is the significant main impetus influencing photosynthetic movement, yet in addition leaf temperature, leaf water status, and numerous others. because nobody configuration rules in all conditions. The geometrically dictated by only two parameters: spreading edge and branch length. are undoubtedly multi-fractals and have consistently pulled in mathematicians for plant shape and development models. In trees, the leaves on the outside of the tree shelter contrast from those inside the tree covering. The outside leaves are alluded to as "sun leaves," while the inside leaves are "conceal leaves." These leaves have contrasts fit as a fiddle, interior life structures, and science that make an interpretation of specific capacities to utilize various types of sun based radiation successfully. Sun leaves are normally littler, progressively prolong, and thicker than concealed leaves, with more layers of chlorophyll-containing tissues and increasingly broad inside vascular frameworks. It is believed that sun leaves are better adjusted to catch and utilize direct 


\section{International Journal of Engineering Applied Sciences and Technology, 2020 Vol. 5, Issue 2, ISSN No. 2455-2143, Pages 217-221 \\ Published Online June 2020 in IJEAST (http://www.ijeast.com)}

sunlight based radiation (when it isn't too exceptional to even think about causing heat and different pressure related harm). Their lengthened shape is likewise connected with more significant levels of sunlight based radiation arriving at the inward covering where the shade leaves are found. Shade leaves appear to successfully utilize diffuse sun oriented radiation, which arrives at the internal shelter in the wake of having been dissipated by different items, similar to the external sun leaves, in the way of direct light. Shade leaves can likewise be found on the outside shelter as an afterthought that faces from the overarching sun. The leaf photosynthesis rate relies upon the biochemical limits of the leaf just as a light appropriation on the leaf surface. The dispersion and course of action of leaves inside a tree can firmly change the light reaping effectiveness. The all-out leaf zone bolstered by a given plant is the most essential auxiliary property that influences the division of consumed radiation. As the sunlight based tendency point diminishes from the equator to higher scopes, trees with contrasting stature to width proportion have inalienably changing efficiencies of light capture. Every single such variety to augment the immediate light capture attempt by the whole tree.

A leaf must "catch" sunlight, water from inside and carbon dioxide from the encompassing air by means of pores for photosynthesis. The leaf photosynthesis, at that point, is influenced by these exercises in careful control: enough daylight and carbon dioxide to run photosynthesis, yet not all that much-related warmth retention or water misfortune. The leaves found on the trees of the Nature Trail are either expensive and level or needle-moulded which appropriate to its climatic conditions for cautious preservation of water and most extreme daylight retention is a significant endurance system. A significant result of variety in a leaf design, its shape and size is the general light gathering and the proficiency factor for photosynthesis.

\section{SOlar POWER TreE}

Nature motivation centres around profoundly requested little bent surface geometry which follows the most extreme snare of daylight and water protection through photosynthesis. Likewise between a sun oriented cell and a tree is that both attempt to catch the most extreme sunlight based energy with least water misfortune or warmth age. At present utilization of $\mathrm{PV}$, frameworks increment quickly because of ceaseless decrease in costs of sun based cells. Still, there are a few obstacles for the appropriation of this innovation like low productivity and enormous space necessities. Different methodologies [6] have been done to improve sun oriented energy factors, for example, low ingestion coefficient, heat loss, reflection coefficient and so forth, The charge bearer age and conduction impact the light conversion proficiency of a sunlight based cell. This is a roundabout way that relies upon the quantity of sun-powered cells introduced and their energy change limit.

Recently inspired by natural heat-trapping technology photosynthesis, a new concept solar tree is introduced by many researchers $[3,4]$. Nature centres around profoundly requested little bent surface geometry like tree geometry. So a gathering of cells which are littler might be prolonged, a little bent and have a multilayer structure. The new idea- Solar Tree is a natural venture, an environmental model, a counterfeit sun based structure that seems as though sculptural trees exist from little scope (size of a bonsai tree) to enormous scope (about the size of a breeze turbine) power plant. In sun based trees, PV boards or cells are orchestrated in a Fibonacci arrangement design rather than leaves. It is an independent unit that produces green energy and provides a place of comfort and energy for a wide variety of services.

The structure is a ground-mounted solar system with a pole that supports many individual panels up in the air. The performance of sunlight based trees vary and they have been intended to give the capacity to face various situations. Sunlight based tree has remarkable properties as far as structure and multi-edge direction parameters. By utilizing the tallness parameter, the sun-powered tree requires less space utilization which can lessen the installation cost. Such a mix of the sun oriented cell should be possible on common trees to lessen the expense of mounting. Contrasted with the customary sun based PV homestead which is situated a solitary way, the multi-edge direction parameter from the sun based tree boards yields the potential ability to retain higher daylight force prompting higher yield vitality. Simultaneously, this parameter disposes of the need to introduce a sun oriented tracker which can decrease the operational expense. To build the assortment Generation of 1MW force from PV module framework requires the space of around 5-6 Acres for lodging the boards as it whereas a solar tree would take just $1 \%$ of the land territory in contrast with general PV lodging. So it is appropriate for places where there is a shortage of land. It can likewise be bolted at any situation to withstand the wind pressure because of substantial tempest influencing over the fundamental shaft/trunk. The boards will be normally looking with least block from different towards the sun at an edge as required so they can gather the greatest sun-powered energy in a daytime.

Sun-powered trees are actually a functional answer for household use. In this setting utilization of PV innovation for the local necessity as the sun oriented tree is the acceptable option when contrasted with traditional level or housetop mounting PV panels. Solar trees can be introduced close, before the house or on a porch, where there is no concealing consistently. To build assortment efficiency, the nanowire cell can be used rather than present PV cells. The Nanowire cells can gather multiple times progressively sun based vitality 
when contrasted with regular cells. The idea of nano leaf is a plan to expand the different types of energies, for example, sunlight based, warm and piezoelectric force by stalks, branches, and twigs of a tree.

Solar trees are really a practical solution for domestic use. In this context use of Photovoltaic technology for the domestic applications in the form of the solar tree is the good alternative when compared with conventional rooftop mountings. Solar trees can be installed in front of the houses, at the terrace, in streets or where there is no shading throughout the day.

\section{Minimization OF SOlar ENERGy LOSS}

Sunlight increases temperature which affects photovoltaic conversion. This does not significantly affect the light generated current of the cell but reverse saturation current increases exponentially with temperature and also reduces open circuit voltage with temperature. Furthermore, it has been experimentally observed that temperature drops the efficiency of solar cell. It is imperative to reduce the heat build-up in the cell in order to improve conversion efficiencies. Recently, it is seen that solar tree energy conversion efficiency increases through minimizing the chlorophyll antennae [6]. Similarly to keep the heating impact of a solar cell low, various approaches are possible. The approaches are possible such as use of multispectral cells for low heat production, reduction in energy loss through current loss and the use of thermal generators for energy harvesting. The reduction in size expected a reduction in current with less utilization time. Recent quantum level technology developed tuneable band gaps across a wide range of energy levels by changing their size, whereas the bandgap is fixed by the choice of bulk materials. This reduces semiconducting particles below the size of the exciton Bohr radius. The quantum nature of existing particles set electron energies finite alike energies in an atom. The developed Quantum dots methodology is referred to as artificial atoms and energy levels are tuneable by changing their size, which in turn defines the bandgap [1]. The dots can be grown over a range of sizes, allowing them to express a variety of band gaps without changing the construction techniques. This makes it attractive for multi-junction solar cells and use of a variety of materials to improve efficiency by harvesting multiple parts of the solar spectrum. To increase the collecting efficiency of cell, the nanowire cells can be used instead of present Photovoltaic cells. The Nanowire cell can collect 15 times more solar energy as compared to conventional cells [1].

\section{FleXible Solar TREE}

From the previous section, it is found that the natural light conversion technology photosynthesis is similar to solar cell technologies. Rather the natural conversion technology is found higher than solar cell light conversion technology $[3,6]$. These natural processes suggest various different possible solutions to these energy losses in a solar cell. The laws of science put some limits on such power conversion, but also leave some scope for improvement through multiple loss minimizations. Section II shows that sun spectrum absorption varies with nature and configuration of leaves, so give an idea of multi-spectrum material for solar cell construction. Also leaves shape, size effect light inclination and efficiency for light energy conversion. This inspiration focuses on small curved surface geometry to capture more sunlight. The multilevel solar cell - tandem cell can be fabricated to drastically reduce the transmission losses. The efficiency of natural photosynthesis also depends on water evaporations; similarly, heat dissipation play is a key factor in solar cell light conversion. .From the previous section, it is also clear that scaling down also affects efficiency drastically.

All these ideas include a modified version of the solar tree which is discussed in section II. The advancement of technology has reduced the size of solar panels to a size of 0.6 $\mathrm{x} 2.55$ inches . This is comparable to the size of plant leaves and their individual identity power varies from 0.5 to $4 \mathrm{~W}$. The cumulative power output will be quite high for various
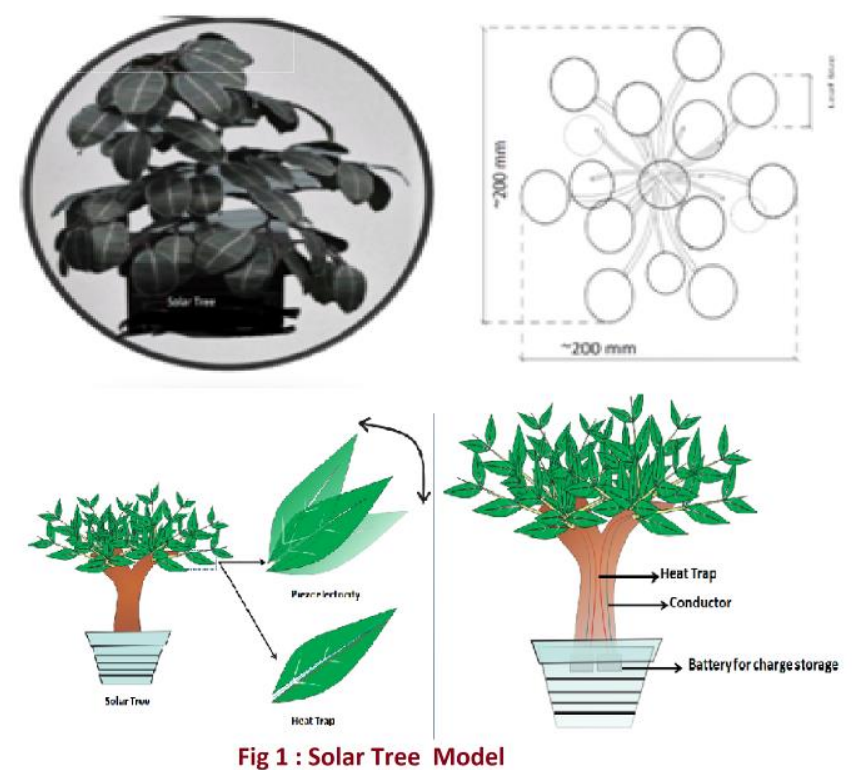

domestic applications. The concept of nano solar material solar tree leaf that would maximize the light conversion $[1,5]$. In order to trap maximum solar energy, the use of quantum wire PV boards is orchestrated in a Fibonacci arrangement design. One such plant pot size is proposed is shown in figure1. In this novel solar tree design, multispectral cell junctions can be fabricated to drastically reduce the transmission losses. On top of the leaves, the anti-reflective coating can be used to reduce reflection losses. Leaves are the independent unit that produces green energy and provides a place of comfort and energy for a wide variety of services. This kind of texturing of the rear surface will not be required 


\section{International Journal of Engineering Applied Sciences and Technology, 2020 \\ Vol. 5, Issue 2, ISSN No. 2455-2143, Pages 217-221 \\ Published Online June 2020 in IJEAST (http://www.ijeast.com)}

to achieve total internal reflection as the surface of flexible materials is already rough.

The leaf photosynthesis is also influenced by these exercises in careful control of carbon dioxide or too much-related water retention. In photosynthesis, this problem of heat generation is solved by molecules energy transfer to another until it settles in a molecule that can store energy for a long time. The conductive branches of photovoltaic solar trees can trap excited electrons into a battery for storage. This leaf-like structure acts as a thermionic generator thereby leading to the enhanced sensitivity. This design ensures the enhanced performance of a solar cell as a reduction in heat-up leads to higher efficiency. In addition to this the flexible nature of leaves also helps in harvesting wind energy. The various forms of energies such as solar, thermal and piezoelectric power by stalks, branches, and twigs of a tree. The amount of energy generation varies with the size/shape of leaves, the number of leaves etc. The flexible nature of coated piezoelectric solar cells helps in harnessing the wind energy throughout day and night. A significant amount of wind power is also generated due to wind-generated movement of small light weighted leaves. The heat energy can be converted simultaneously into electric energy using thermal converters. the energy of radiations. The various designs of flexible trees can be possible depending on geographical conditions and demands. The synchronization of solar energy with heat energy and wind energy supply continuous supply of energy even at night or when the sun is covered with clouds.

\section{CONCLUSIONS}

The The sunlight based tree idea is effective to satisfy the expanding energy demands of the individuals, sparing area, and ought to be executed to give power without the issue of intensity cut-off. The concept of nano leaf is an idea to maximize the various forms of energies such as solar, thermal and piezoelectric power by stalks, branches, and twigs of a tree.

All this energy waste harvesting converts it into grid power. The adaptable nature and nano-size leaf fit it into a beautiful small size pot plant and a valuable device. This superb plant that requires no watering and sustains. The beginning venture cost of the sun based tree is likewise equivalent to the same limit PV frameworks as other framework parts are similar. The restitution time of proposed framework is by all accounts high yet because of persistent increment in the expense of network force and decrease in the expense of PV cell because of mechanical progression long recompense period can be compensated. The same photosynthesis move configuration can be reached out to different areas and applications including higher energy utilizations. At the last, Solar Tree front line eco-perfect innovation and should work in agreement with common trees.

\section{REFERENCE}

[1] Baskoutas, Sotirios; Terzis, Andreas F. (2006). "Sizedependent band gap of colloidal quantum dots". Journal of Applied Physics. 99 (1): 013708-0137084.Bibcode:2006JAP....99a3708B. doi:10.1063/1.2158502

[2] Berny S. and others (2015), Solar Trees: First Large-Scale Demonstration of Fully Solution Coated, Semitransparent, Flexible Organic Photovoltaic Modules, , Advance Science, December 2015 , https://doi.org/10.1002/advs.201500342

[3] C. Bhuvaneswari, R Rajeswari, C.Kalaiarasan and K.M.S. Muthukumararajaguru (2013), Idea to Design a Solar Tree Using Nanowire Solar Cell International Journal of Scientific and Research Publications, Volume 3, Issue 12, December 20131 ISSN 2250-3153.

[4] Economictimes (2019), Mini Solar Panel: Ideal power source for small devices/portable appliances, Read more at: Economictimes.indiatimes.com

[5] Kumar M. and other (2016), Traffic driven wind energy generator, Technology Letters, Vol.3 No.1, p10-13 (ISSN: 2348-8131).

[6] Melis A. (2009), Plant and Microbial Biology, University of California, Berkeley, CA 94720-3102, USA, Plant Science 177, p 272-280. Gupta S., and others (2015), "The benefits and applications of Solar Tree With Natural Beauty Of Trees", SSRG International Journal of Electrical and Electronics Engineering, Issue April 2015 Vol-4 Issue-2 2018 IJARIIE-ISSN(O)-2395-4396 7653.

[7] Srinivas (2016), A P R, "Design and Development of a SOLAR TREE", International Journal of Scientific \& Engineering Research, Volume 7, Issue 10, October-2016 ISSN 2229-5518 